\title{
Emprego da nimodipina (oxigen) como protetor cerebral na cirurgia de revascularização do miocárdio com circulaçẳo extracorpórea em pacientes idosos
}

\author{
Ricardo MANRIQUE*, Ricardo PAVANELLO*, Hélio M. de MAGALHÃES*
}

RBCCV $44205-316$

\begin{abstract}
Manrique R, Pavanello R, Magalhães HM - Emprego da nimodipina (oxigen) como protetor cerebral na cirurgia de revascularização do miocárdio com circulação extracorpórea em pacientes idosos. Rev Bras Cir Cardiovasc 1996; 11 (4): 248-58.
\end{abstract}

RESUMO: Aproximadamente $5 \%$ dos pacientes submetidos a cirurgia cardiaca com auxilio da circulaçăo extracorpórea apresentaram problemas neurológicos. Avaliando funçōes neuropsiquicas, as alterações atingem de $50 \%$ a $70 \%$ dos casos. Os idosos são mais vulneráveis; nestes, a frequeência de acidente vascular cerebral (AVC) aproxima-se dos $9 \%$. Considerando que a populaçāo envelhece a um ritmo acelerado e que o coronariopata freqũentemente passa dos 65 anos de idade, é fundamental pesquisar meios profiláticos para diminuir esta incidência. Este é um estudo piloto, duplo cego, randomizado e controlado com 64 pacientes, 30 noGrupo nimodipina e 34 no Grupo placebo. As variáveis demográficas e diagnósticas pré-operatórias foram homogêneas, com exceção da incidência de isquemia cerebral transitória. O Grupo nimodipina, mesmo com programação cirúrgica e evolução intra e pós-operatória mais complicadas, apresentou menor número de casos neurológicos. NoGrupo nimodipina foram constatados 3 casos de confusão mental e, no Grupo placebo, além de 3 casos de confusão mental, foram diagnosticados mais 2 casos de sonolência e 1 AVC isquêmico com seqüela. No total, foram $3(10 \%)$ casos em 30 pacientes no Grupo nimodipina, e 6 $(17,64 \%)$ em 34 pacientes no Grupo placebo, caracterizando uma redução de $76,4 \%$. LEGAULT et al. (15) relatam uma elevada mortalidade de pacientes em uso de nimodipina, quando operados para troca valvar, causada, principalmente, por hemorragia. Nós não encontramos esta correlação. A mortalidade hospitalar é similar em ambos os grupos ( 1 paciente em cada) e o sangramento não é estatisticamente diferente ( 1 caso de hemorragia importante no Grupo nimodipina). No seguimento até 41 meses, a mortalidade no Grupo placebo foi maior (4 pacientes) que no Grupo nimodipina (1 paciente).

DESCRITORES: Revascularização miocárdica. Isquemia cerebral, prevenção e controle. Confusão, prevenção e controle. Nimodipina, uso terapêutico. Nimodipina administração e dosagem. Revascularização miocárdica, mortalidade. Circulação extracorpórea, efeitos adversos. Método duplo-cego.

\section{INTRODUÇÃO}

Estudos prospectivos realizados em pacientes submetidos a cirurgia cardiaca com auxilio de circulação extracorpórea assinalam uma incidência de $4,8 \%$ a $5,2 \%$ de problemas neurológicos, indepen- dentemente de idade, antecedentes pré-mórbidos e tipo de operação (1).

As alterações neuro-psiquicas atingem de $50 \%$ a $70 \%$ dos casos $(2,3)$.

Uma variável independente é a idade; assim, a

Trabalho realizado no Hospital do Coração da Associação do Sanatório Sírio e no Instituto Dante Pazzanese de Cardiologia. São Paulo, SP, Brasil. Recebido para publicação em novembro de 1996.

*Do Hospital do Coração da Associação Sirio e do Instituto Dante Pazzanese de Cardiologia.

Endereço para correspondência: Rua Desembargador Eliseu Guilherme, 123. São Paulo, SP, Brasil. CEP: 04004-030. Tel: (011) 887-6611. 
Manrique R, Pavanello R, Magalhāes H M - Emprego da nimodipina (oxigen) como protetor cerebral na cirurgia de revascularização do miocárdio com circulação extracorpórea em pacientes idosos. Rev Bras Cir Cardiovasc 1996; 11 (4):248-58 .

freqüência do acidente vascular cerebral (AVC) em pacientes com mais de 75 anos de idade aproximase dos $9 \%$.

WEINTRAUB et al. (4), em estudo multicêntrico, demonstraram, nesta população, um aumento da permanência na UTI, com intubação endotraqueal e assistência respiratória prolongadas, condições que aumentam o risco de infecção respiratória e o custo hospitalar.

Do ponto de vista social, há preocupação, porque, em uma importante parcela destes pacientes, se estabelecem seqüelas definitivas, que eliminam os benefícios da correção cirúrgica e levam a uma situação crônica, com perda da qualidade de vida. A família e a sociedade arcam com o ônus de uma terapia continuada, sem a esperança de resultados satisfatórios.

Considerando que a população envelhece a um ritmo acelerado e que o paciente coronariano freqüentemente passa o limite dos 65 anos, é fundamental pesquisar meios profiláticos que diminuam esta incidência.

Novas drogas são promissoras na profilaxia do dano cerebral induzido pela circulação extracorpórea. Esta afirmativa beseia-se em experiências de neuroproteção com tiopental, gangliósidos GM1, prostaciclinas, isofluorane, perfluorocarbono, polietileno-glicol-superdismutase e, ultimamente, nimodipina. Segundo MILLS (1), a nomodipina constitui a mais promissora droga atualmente disponivel na prevenção do dano cerebral.

Apesar da evidência da morbídade neurológica que acomanha a cirurgia cardiaca em pacientes idosos, chama atenção, na pesquisa bibliográfica, a ausência de estudos sobre a profilaxia das alterações neurológicas.

\section{CARACTERÍSTICAS DO ESTUDO}

Este trabalho foi planejado como estudo piloto, duplo cego, randomizado e controlado.

As amostras vero e placebo foram cedidas pelos Laboratórios Biosintética.

A pesquisa foi desenhada para realizar um minimo de 60 casos, 30 em cada grupo.

O objetivo é desenhar um protocolo de profilaxia do dano cerebral em pacientes idosos que serão submetidos a cirurgia cardíaca com auxillio da circulação extracorpórea.

\section{CASUISTICA E MÉTODOS}

\section{Casuística}

De acordo com o protocolo, as condições fundamentais eram: idade superior a 65 anos e indicação de revascularização do miocárdio com circulação extracorpórea.

\section{Critérios de Inclusão}

Pacientes de ambos os sexos, com 65 ou mais anos de idade, portadores de coronariopatia obstrutiva, passível de revascularização miocárdica. $\mathrm{Na}$ concomitância de doença circulatória cerebral, além da avaliação clínica neurológica, foram realizadas angiografia digital e tomografia computadorizada ou ressonância nuclear magnética, para definir lesão orgânica parenquimal. Pacientes com um único evento de isquemia cerebral transitória, que tenham permanecido estáveis nos últimos 6 meses, seguiram o mesmo protocolo. Em ambas as circunstâncias, se confirmada a ausência de lesão orgânica parenquimal, o paciente foi incluído.

\section{Critérios de Exclusão}

Bradicardia, com freqüência cardíaca igual ou menor que 50 batimentos por minuto; fração de ejeção menor que $40 \%$; pressão sistólica menor que 90 $\mathrm{mmHg}$; insuficiência renal (creatinina maior que 1,6 $\mathrm{mg} / \mathrm{dl}$ ); insuficiência hepática de qualquer grau; lesão orgânica neurológica; alteração psíquica; diabetes melito descompensada (glicemia de jejum maior de $180 \mathrm{mg} / \mathrm{dl}$ ), medição associada proibida pelo protocolo, conforme Tabela 1. Contra-indicação primária ao uso de antagonista de cálcio em geral.

\section{Protocolo}

O protocolo foi dividido em cinco ítens:

- Dados gerais de identificação

Antecedentes familiares e pessoais

TABELA 1

RELAÇÃO DE DROGAS NĀO PERMITIDAS

\begin{tabular}{llll}
\hline Cinarizina & Flunarizina & Benciclano & Nicergolina \\
Buflomedil & Natridofurilo & Vinacamina & Piracetam \\
Pirabedil & Carbamezipina & Fenobarbital & Finitoina \\
Valproato & Ginko Biloba & Mesilato de Codergocrina \\
Ácido Nicotínico com Papaverina & \\
\hline
\end{tabular}


Manrique R, Pavanello R, Magalhāes HM - Emprego da nimodipina (oxigen) como protetor cerebral na cirurgia de revascularização do miocárdio com circulação extracorpórea em pacientes idosos. Rev Bras Cir Cardiovasc 1996; 11 (4):248-58 .

- História, exame clínico, cardiológico e neurológico pré-operatório

- Características e evolução do intra-operatório

- Evolução clínica, cardiológica, neurológica e laboratorial pós-operatória

O controle laboratorial de rotina foi registrado em folha à parte.

Especial atenção foi prestada a efeitos colaterais, que foram coletados em dados específicos.

\section{Esquema Terapêutico}

Foram infundidos $50 \mathrm{mg}$ de nimodipina (Oxigen)/ dia ou placebo, desde o pré-operatório imediato, usando uma bomba de infusão contínua e sempre com o equipo de infusão específico para nimodipina. A administração prosseguiu no intra-operatório, com o cuidado de infundir diretamente na veia do paciente (não na máquina de circulação extracorpórea). Durante a permanência na UTI, continuou a administração por via endovenosa na mesma dose. $\mathrm{Na}$ enfermaria, passamos para a via oral, com $90 \mathrm{mg} /$ dia divididos em três tomadas, dose que se manteve até a alta hospitalar.

\section{Pontos Finais}

Como pontos finais de observação, foram determinados:

- mortalidade geral

- mortalidade neurológica

- morbidade neurológica

- permanência hospitalar
Considerou-se fracasso terapêutico a presença de sintomas ou sinais neurológicos durante a evolução hospitalar.

\section{Avaliação Estatística}

A amostra foi avaliada usando-se o princípio do intention to treat. Os procedimentos estatísticos utilizados foram: Teste T de Student e o Qui quadrado bilaterais. $O$ nível de significância foi definido por um "p" bilateral igual ou menor que 0,025 , ou seja, por um intervalo de confiança de $95 \%$.

\section{RESULTADOS}

Foram estudados 64 pacientes, 30 no Grupo nimodipina e $34^{*}$ no Grupo placebo, sendo 21 homens e 9 mulheres no Grupo nimodipina e 25 homens e 9 mulheres no Grupo placebo.

A análise foi realizada em quatro etapas:

- Na primeira, foram comparadas as características demográficas e clínicas pre-operatórias.

- Na segunda, as condições intra-operatórias.

- Na terceira, a evolução pós-operatória até a alta hospitalar ou óbito do paciente.

- Na quarta, a análise dos subgrupos sintomáticos neurológicos.

$\mathrm{Na}$ Tabela 2, encontramos os dados referentes às características demográficas e diagnósticas préoperatórias:

Todos os parâmetros analisados mostraram-se semelhantes nos dois grupos; a única diferença estatisticamente significativa foi a maior incidência de

TABELA 2

DADOS DEMOGRÁFICOS E CLINNICOS PRÉ-OPERATÓRIOS

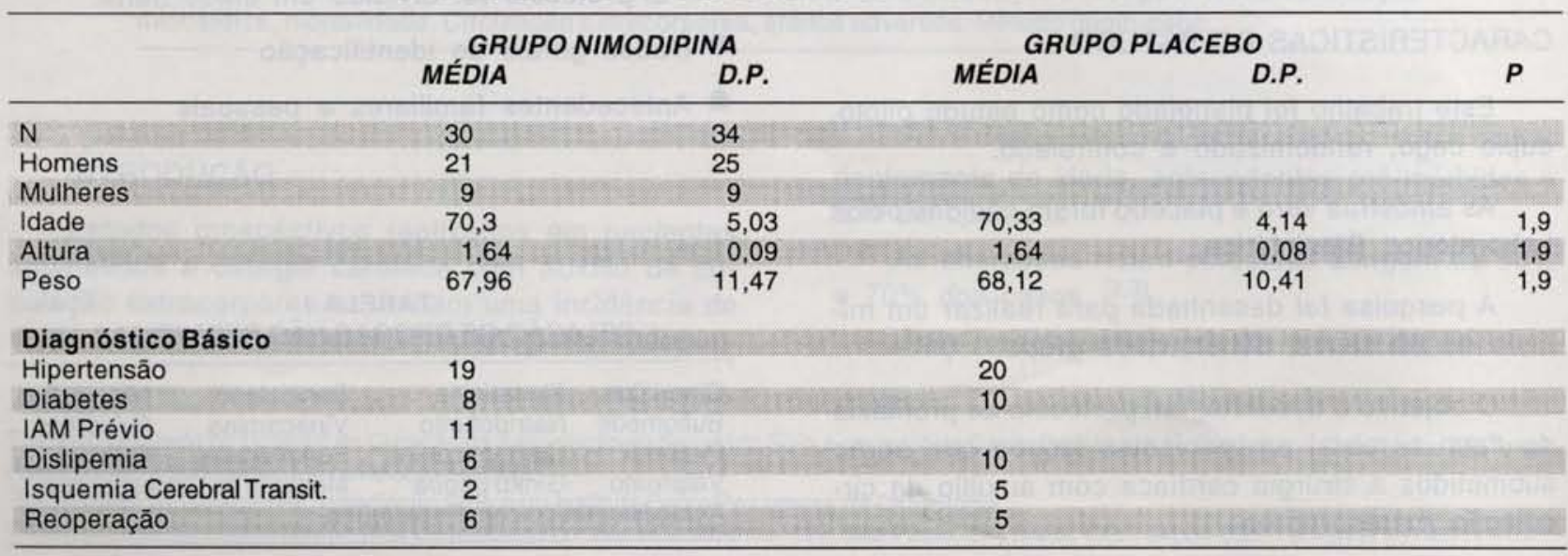


Manrique R, Pavanello R, Magalhães H M - Emprego da nimodipina (oxigen) como protetor cerebral na cirurgia de revascularização do miocárdio com circulação extracorpórea em pacientes idosos. Rev Bras Cir Cardiovasc 1996; 11 (4):248-58

TABELA 3

DISTRIBUIÇÃO DAS LESÓES CORONARIANAS

\begin{tabular}{lcccc}
\hline LESÖES CORONARIANAS & DISCRETA & MODERADA & SEVERA & OCLUIDA \\
\hline Grupo Nimodipina & & & & 5 \\
DA & 1 & 2 & 19 & 5 \\
CX & 2 & 10 & 4 & 9 \\
CD & 2 & 3 & 8 & 5 \\
GrupoPlacebo & 1 & 4 & 21 & 2 \\
DA & 1 & 10 & 15 & 8 \\
CX & 4 & 8 & 8 & \\
CD & & & \\
\hline
\end{tabular}

TABELA 4

CARACTERISTICAS DAS LESÕES CAROTÍDEAS NOS GRUPOS NIMODIPINA E PLACEBO

\begin{tabular}{ccccc} 
Lesōes de Carótidas & & & & \\
Carótida Direita & Discreta & Moderada & Severa & Ocluída \\
Grupo Nimodipina & 6 & 2 & 1 & Ocluída \\
Grupo Placebo & 4 & 4 & Severa & \\
Carótida Esquerda & Discreta & Moderada & 2 & \\
Grupo Nimodipina & 7 & 1 & 0 & \\
Grupo Placebo & 3 & 6 & & \\
\hline
\end{tabular}

TABELA 5

PROCEDIMENTOS CIRÚRGICOS

\begin{tabular}{lcc}
\hline $\begin{array}{l}\text { PROCEDIMENTO } \\
\text { CIRÚRGICO }\end{array}$ & $\begin{array}{c}\text { GRUPO } \\
\text { NIMODIPINA }\end{array}$ & $\begin{array}{c}\text { GRUPO } \\
\text { PLACEBO }\end{array}$ \\
\hline Revascularização & & \\
do Miocárdio (RM) & 21 & 31 \\
RM e Aneurisma VE & 2 & 0 \\
RM e Carótida & 3 & 1 \\
RM e Outras & 4 & 2 \\
\hline
\end{tabular}

isquemia cerebral transitória, de qualquer origem, no Grupo placebo (5) comparado com o Grupo nimodipina (2).

$\mathrm{Na}$ Tabela 3, encontramos a distribuição das lesões coronarianas. Dado o número pequeno de observações não foi possivel avaliar estatisticamente esta distribuição, porém chama a atenção a semelhança, e como única discrepância, a freqüência maior das lesões severas de circunflexa no grupo placebo.

As lesões carotídeas foram mais freqüentes no Grupo nimodipina, mas, se eliminarmos as lesōes discretas, as lesões moderadas e severas são totalmente equivalentes (Tabela 4 ).

A avaliação intra-operatória qualifica o tipo de procedimento cirúrgico proposto e a evolução clínica e a laboratorial no centro cirúrgico. Por definição, todos os pacientes foram submetidos a uma revascularização do miocárdio.

Na Tabela 5, observamos que, no Grupo nimodipina, foi realizado maior número de procedimentos complexos (9), contra só 3 no Grupo placebo.

As variáveis de controle clínico e laboratorial intra-operatórios, assim como o tempo de anestesia e de circulação extracorpórea, foram semelhantes nos dois grupos (Tabela 6).

A evolução na UTI do Grupo nimodipina foi mais tumultuada, com 2 casos de IAM no pós-operatório, que apresentaram síndrome de baixo débito, com óbito. No Grupo placebo, na enfermaria, observou-se 1 AVC isquêmico, que evoluiu com seqüela, e outro paciente foi a óbito (Tabela 7).

$\mathrm{Na}$ avaliação neurológica, observamos 3 casos sintomáticos no Grupo nimodipina contra 6 no Grupo placebo. Em todos os casos os sintomas neurológicos foram reversíveis, menos no paciente com AVCi. Os dados se encontram na Tabela 8.

Esta análise nos indica uma redução de $76,4 \%$ na incidência de sintomas neurológicos no Grupo nimodipina contra $\circ$ Grupo placebo.

Alguns dados de importância aparecem quando estudamos a pequena população de 9 pacientes com sintomas neurológicos.

Seguimos a mesma metodologia adotada no procedimento principal. 
Manrique R, Pavanello R, Magalhães HM - Emprego da nimodipina (oxigen) como protetor cerebral na cirurgia de revascularização do miocárdio com circulação extracorpórea em pacientes idosos. Rev Bras Cir Cardiovasc 1996; 11 (4):248-58 .

TABELA 6

EVOLUÇÃOINTRA-OPERATÓRIA

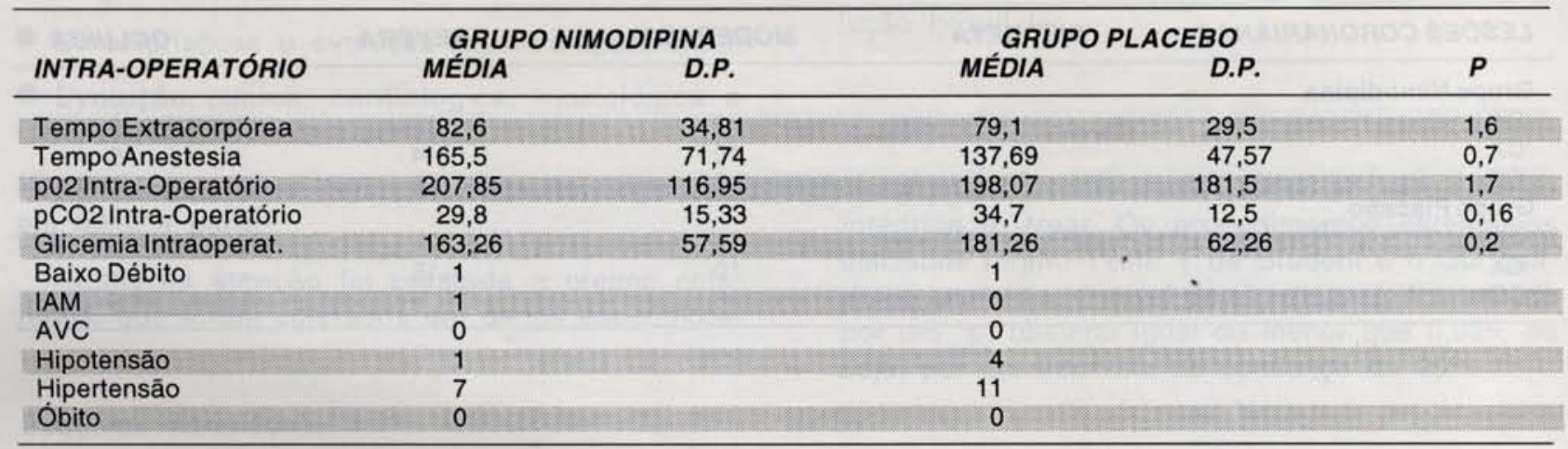

TABELA 7

EVOLUÇĀOPÓS-OPERATÓRIA

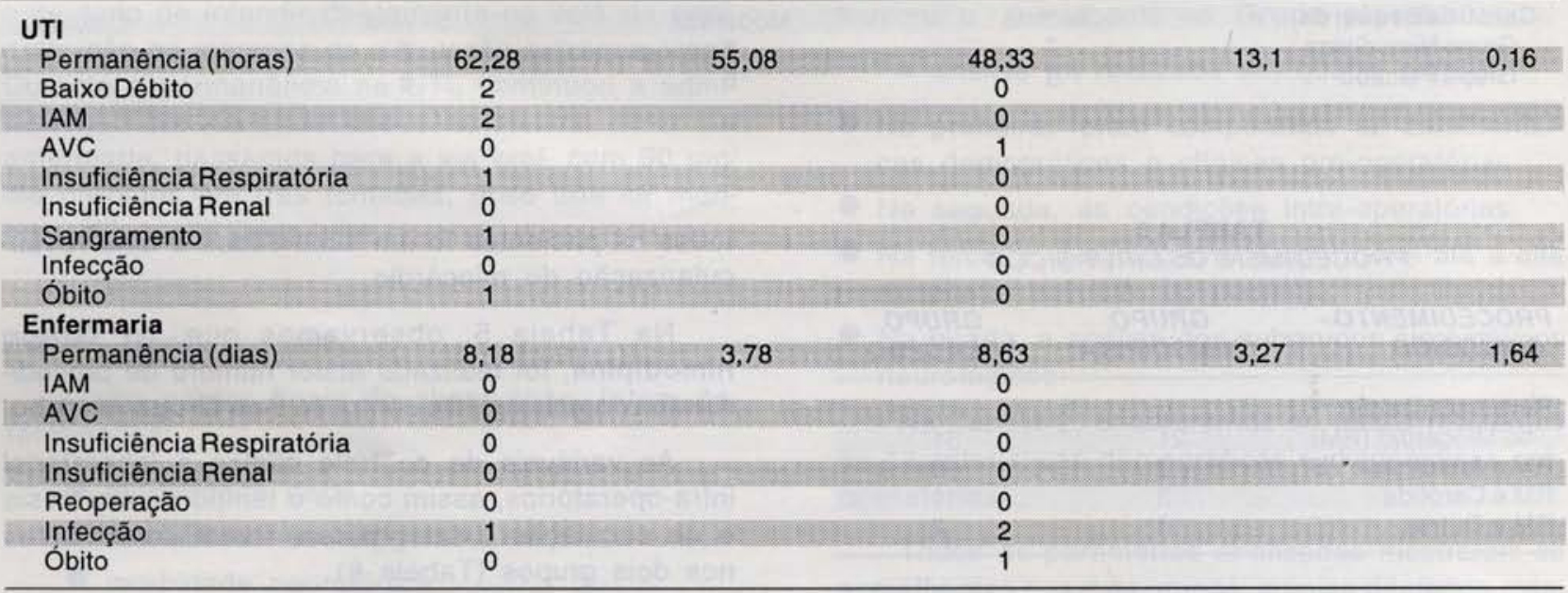

TABELA 8

EVOLUÇĀO NEUROLÓGICA

\begin{tabular}{lcc}
\hline $\begin{array}{l}\text { COMPLICAÇŌES } \\
\text { NEUROLOGICAS }\end{array}$ & $\begin{array}{c}\text { GRUPO } \\
\text { NIMODIPINA }\end{array}$ & $\begin{array}{c}\text { GRUPO } \\
\text { PLACEBO }\end{array}$ \\
\hline Confusão Mental & 3 & 3 \\
Sonolência & 0 & 2 \\
AVC & 0 & 1 \\
Coma & 0 & 0 \\
\hline Total de Eventos & 3 & 6 \\
\hline
\end{tabular}

Os parâmetros demográficos e diagnósticos clínicos não são estatisticamente diferentes entre os grupos, com exceção da isquemia cerebral transitória (Tabela 9).

As discrepâncias aparecem quando analisamos os tempos de anestesia e de circulação extra- corpórea, nos quais o "p" é de 0,008 para o tempo de anestesia e de 0,009 para o tempo de extracorpórea, em relação a um maior tempo anestésico e de circulação extracorpórea no Grupo nimodipina, claramente relacionado com a maior complexidade dos procedimentos cirúrgicos (Tabela 10).

Numa análise dos tipos de operação realizados observamos que, no Grupo nimodipina, as manifestações neurológicas reversíveis se apresentaram em pacientes com operaçōes complexas com tempo cirúrgico prolongado, enquanto que, no Grupo placebo, todos os casos neurológicos foram de revascularização isolada com tempos de anestesia e de circulação extracorpórea na média (Tabela 11).

As diferenças da evolução se apresentam também na permanência em UTI e enfermaria.

Os pacientes neurológicos, do Grupo nimodi- 
Manrique R, Pavanello R, Magalhães H M - Emprego da nimodipina (oxigen) como protetor cerebral na cirurgia de revascularização do miocárdio com circulação extracorpórea em pacientes idosos. Rev Bras Cir Cardiovasc 1996;11 (4):248-58.

TABELA 9

DADOS DEMOGRÁFICOS E DIAGNÓSTICOS DOS SUBGRUPOS SINTOMÁTICOS

\begin{tabular}{|c|c|c|c|c|c|}
\hline \multirow{2}{*}{ 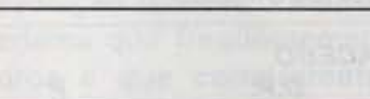 } & \multicolumn{2}{|c|}{ GRUPO NIMODIPINA } & \multicolumn{2}{|c|}{ GRUPO PLACEBO } & \multirow[b]{2}{*}{$P$} \\
\hline & MÉDIA & D.P. & MÉDIA & D.P. & \\
\hline $\mathrm{N}$ & 3 & & 6 & & \\
\hline Homens & 3 & & 6 & & \\
\hline Mulheres & 0 & & 0 & & \\
\hline Idade & 68,75 & 5,19 & 74,75 & 6,13 & 0,181 \\
\hline Altura & 1,72 & 0,09 & 1,65 & 0,07 & 0,08 \\
\hline Peso & 73,75 & 10,81 & 64,75 & 7,37 & 0,168 \\
\hline \multicolumn{6}{|l|}{ Diagnóstico Básico } \\
\hline Hipertensāo & 3 & & 3 & & \\
\hline Diabetes & 0 & & 0 & & \\
\hline IAM Prévio & 3 & & 3 & & \\
\hline Dislipemia & 1 & & 0 & & \\
\hline Isquemia Cerebral Trans. & 0 & & 3 & $2=$ & \\
\hline Reoperaçāo & 2 & & 1 & & \\
\hline
\end{tabular}

TABELA 10

TEMPOS COMPARATIVOS DE CIRCULAÇĀO EXTRACORPÓREA E ANESTESIA NOS SUBGRUPOS DE PACIENTES COM EVENTOS NEUROLÓGICOS

\begin{tabular}{lccc}
\hline & GRUPO NIMODIPINA & GRUPO PLACEBO & P \\
\hline Tempo de Extracorpórea & $127+/-46,8$ minutos & $61,71+/-15,13$ & 0,008 \\
Tempo de Anestesia & $257+/-(83,59)$ & $130,85+/-54,33$ & 0,009 \\
\hline
\end{tabular}

TABELA 11

TIPO DE CIRURGIA NOS SUBGRUPOS SINTOMÁTICOSNEUROLÓGICOS

\begin{tabular}{lcc}
\hline $\begin{array}{l}\text { PROCEDIMENTO } \\
\text { CIRURGICO }\end{array}$ & $\begin{array}{c}\text { GRUPO } \\
\text { NIMODIPINA }\end{array}$ & $\begin{array}{c}\text { GRUPO } \\
\text { PLACEBO }\end{array}$ \\
\hline Revascularização & & \\
do Miocárdio & 0 & 6 \\
RM e Aneurisma do VE & 2 & 0 \\
RM e Carótida & 1 & 0 \\
\hline
\end{tabular}

pina, apresentaram quadros clínicos mais graves, sendo a permanência em ambas as unidades maior e estatisticamente significativa quando comparada com o Grupo placebo (Tabela 12).

No Grupo nimodipina a média de sangramento foi de $651,38 \mathrm{ml}$, em 48 horas, com limites de confiança de $95 \%+/-232,95 \mathrm{ml}$. No Grupo placebo a média foi de $559,55 \mathrm{ml}$ em 48 horas, com intervalo de confiança de $95 \%+/-108,91 \mathrm{ml}$.

A avaliação estatística pelo teste $T$ de Student foi de um " $p$ " de 0,78 , não alcançando a faixa da significância. (Tabela 13).
$\mathrm{Na}$ análise dos grupos subdivididos por faixas de volume drenado e distribuidas as respectivas freqüências, encontramos os resultados seguintes:

$\mathrm{Na}$ coluna Histórico mostramos a distribuição da drenagem dos pacientes do Hospital do Coração, com operação de revascularização do miocárdio, nos últimos cinco anos (Tabela 14). Comparados com o Grupo nimodipina, observamos que ambos são semelhantes. No Grupo placebo chama a atenção a concentração de pacientes com drenagem de até $750 \mathrm{ml}$.

O controle no pós-operatório tardio foi no minimo de 24 meses e no máximo de 41 meses. A mortalidade dos pacientes ao longo deste período foi de 1 paciente no Grupo nimodipina e 4 no Grupo placebo.

\section{COMENTÁRIOS}

A nimodipina é um bloqueador dos canais de cálcio, lipofílico e capaz de atravessar a barreira hematocerebral. Atua como um potente vasodilatador cerebral e consegue aumentar o fluxo sangüíneo local sem alterar, significativamente, a pressão arterial sistêmica. Apresenta, também, uma ação 
TABELA 12

PERMANENNCIA NA UTI E ENFERMARIA DOS SUBGRUPOS COM SINTOMAS NEUROLÓGICOS

\begin{tabular}{|c|c|c|c|c|c|}
\hline & \multicolumn{2}{|c|}{ GRUPO NIMODIPINA } & \multicolumn{2}{|c|}{ GRUPO PLACEBO } & \multirow[b]{2}{*}{$P$} \\
\hline & MÉDIA & D.P. & MÉDIA & D.P. & \\
\hline \multicolumn{6}{|l|}{ UTI } \\
\hline Permanência (horas) & 180,33 & 135,94 & 52 & 13,86 & 0,028 \\
\hline Baixo Débito & 2 & & 1 & & $\sqrt{1}+2$ \\
\hline IAM & 1 & & 0 & 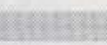 & 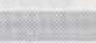 \\
\hline AVC & 0 & & 1 & & 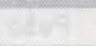 \\
\hline Insuficiência Respiratória & 1 & & 0 & & \\
\hline Insuficiência Renal & 0 & & 1 & & \\
\hline Sangramento & 1 & & 0 & & \\
\hline Infecção & 0 & & 0 & & \\
\hline Óbito & 1 & & 0 & & \\
\hline \multicolumn{6}{|l|}{ Enfermaria } \\
\hline Permanência (dias) & 13,5 & 4,95 & 6,5 & 0,58 & 0,0586 \\
\hline IAM & 0 & & 0 & & \\
\hline AVC & 0 & & 0 & & \\
\hline Insuficiência Respiratória & 0 & & 0 & & \\
\hline Insuficiência Renal & 0 & & 0 & & \\
\hline Reoperação & 0 & 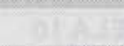 & 0 & & \\
\hline Infecção & 0 & $3=\frac{1}{10}$ & 0 & & \\
\hline Óbito & 0 & & 1 & & \\
\hline
\end{tabular}

TABELA 13

ANÁLISE ESTATISTICA DA DRENAGEM TORÁCICA DOS GRUPOS NIMODIPINA E PLACEBO

\begin{tabular}{lcccc}
\hline$G$ & DRENAGEM & D.P. & TCALCULADO & $P$ \\
\hline Nimodipina & 651.38 & 536.83 & 0.799 & 0.78 \\
Placebo & 550.55 & 342.43 & & \\
\hline
\end{tabular}

TABELA 14

COMPARAÇÃO PERCENTUAL DA DISTRIBUIÇÃO POR VOLUME DE DRENAGEM

\begin{tabular}{lccr}
\hline DRENAGEM & HISTÓRICO & NIMODIPINA & PLACEBO \\
\hline $0.750 \mathrm{ml}$ & $76 \%$ & $23(77 \%)$ & $30(88 \%)$ \\
$751-1000 \mathrm{ml}$ & $14 \%$ & $3(10 \%)$ & $1(3 \%)$ \\
$1001-1500 \mathrm{ml}$ & $6 \%$ & $3(10 \%)$ & $2(6 \%)$ \\
$>1500 \mathrm{ml}$ & $4 \%$ & $1(3 \%)$ & $1(3 \%)$ \\
\hline
\end{tabular}

TABELA 15

MORTALIDADE INTRA-HOSPITALARE TARDIA. ANÁLISE DE CAUSA DE OBITO.

\begin{tabular}{lccc} 
Mortalidade Intra-hospitalar & Tardia & Total \\
Nimodipina & 1 & 1 & 2 \\
Placebo & 1 & 4 & 5 \\
Causa do Óbito & Evento Cardiovascular & Outras \\
Nimodipina & 2 & 0 \\
Placebo & 4 & 1 \\
\hline
\end{tabular}

bioquímica neuronal direta que, possivelmente, caracteriza o mais importante efeito neuroprotetor.

A nimodipina tem um efeito vasodilatador preferencial sobre a contração dos vasos cerebrais, induzida in vitro, por neurotrasmissores e substâncias vasoconstritoras (5).

Existe uma clara evidência de que a nimodipina dilata vasos cerebrais in vitro e vasos da Pia-mater in vivo (6).

Estas referências foram confirmadas com experimentos em babuínos e ratos, demonstrando que o fluxo cerebral aumenta em conseqüência da administração de nimodipina (7).

Quando experimentalmente se produz uma isquemia grave por ligadura da artéria cerebral média com aplicação prévia e contínua de nimodipina, se observa uma redução da lesão isquêmica sem alteração da área necrótica, minimizando o dano histológico e suas conseqüências fisiopatológicas.

O efeito benéfico deve estar relacionado ao aumento do fluxo cerebral e à proteção do neurônio em relação ao metabolismo do cálcio, evitando o efeito deletério da avalanche deste ion no interior da célula nervosa em condiçōes isquêmicas.

Fisiopatologicamente, as fases do fluxo cerebral após um período de completa isquemia experimental são de uma hiperemia que dura de 10 a 15 minutos, seguida por uma fase de hipofluxo de 18 a 48 horas ${ }^{(8)}$. O hipofluxo pós isquemia reduz de 
Manrique R, Pavanello R, Magalhães H M - Emprego da nimodipina (oxigen) como protetor cerebral na cirurgia de revascularização do miocárdio com circulação extracorpórea em pacientes idosos.

Rev Bras Cir Cardiovasc 1996; 11 (4):248-58.

$75 \%$ a $80 \%$ o fluxo cerebral total. O destino e a evolução do tecido neuronal dependem também do edema que freqüentemente acompanha estes quadros e que compromete a microcirculação com capilares no reflow, agravando o quadro isquêmico inicial e explicando o caráter evolutivo desta lesão.

Experimentalmente, tem sido demonstrado, em cachorros, com isquemia induzida por oclusão da aorta e das duas veias cavas por 10 minutos, que a nimodipina consegue reduzir o periodo de hipofluxo pós-isquêmico $(9,10)$. No grupo de cachorros protegidos se infundiam previamente $10 \mu \mathrm{g} / \mathrm{g} / \mathrm{min}$ por 2 horas. O fluxo cerebral destes animais dobrava no período pós-isquêmico em relação ao grupo controle. paralelamente, aumentava, também neste grupo, o consumo de oxigênio pelo tecido cerebral, indicando que não se tratava de um "hiperfluxo de luxo". Esta droga também influencia a evolução, diminuindo a incidência de seqüelas $(11,12)$.

Clinicamente, tem se mostrado eficaz para reduzir a morbi-mortalidade na hemorragia subaracnóidea. Quando aplicado no período de $24 \mathrm{~h}$ a $48 \mathrm{~h}$ após AVC, consegue reduzir a área de infarto e melhorar o prognóstico destes pacientes (5).

FORSMAN et al. ${ }^{(13)}$ relataram menor incidência de distúrbios neuropsíquicos após cirurgia com circulação extracorpórea, quando utilizada a nimodipina desde o pré-operatório.

Na cirurgia cardíaca, o mecanismo fisiopatológico provavelmente está relacionado com a circulação extracorpórea. Esta afirmativa se apoia na baixa freqüencia destas complicações em toracotomias clássicas ou cirurgia cardíaca sem circulação extracorpórea. Pacientes com alteraçōes vasculares da crculação endocraniana caracterizam a populção de risco. Neste casos, a condição não pulsátil do fluxo sangüineo durante a operação, assim como variaçōes pressóricas, contribuem para comprometer a nutrição do tecido cerebral.

A nivel celular, o ingresso em excesso e acúmulo de íons cálcio no citosol contribuem, de modo determinante, para o dano neuronal pós-isquêmico (12).

A nossa proposta de pesquisar a nimodipina como profilático da agressão neuronal isquêmica pós perfusão extracorpórea baseia-se no efeito antagônico do cálcio e na sua capacidade para atravessar a barreira hematocerebral.

No presente estudo, avaliamos 64 pacientes, 4 casos a mais da casuística mínima, para compensar eventuais pacientes excluidos posteriormente.

Não observamos diferenças nas variáveis demográficas, porém fica enfatizada a importância do antecedente de sintomatologia isquêmica cerebral prévia como fator de risco de morbidade neurológi- ca no pós-operatório; assim, dos 5 pacientes do grupo placebo com este antecedente, 3 apresentaram eventos neurológicos. No grupo nimodipina, dos 2 pacientes com o mesmo antecedente, nenhum apresentou complicação neurológica. Isto nos permite sugerir que, pelo menos nos pacientes com antecedente de isquemia cerebral de qualquer origem e, mesmo, sem seqüelas, é recomendável instituir um esquema profilático.

$\mathrm{Na}$ avaliação global das complicações neurológicas no Grupo placebo constatamos, em 34 casos, 3 casos de confusão mental, 2 de sonolência e 1 AVC com seqüelas. No Grupo nimodipina, em 30 pacientes, observamos só 3 casos de confusão mental. A análise primária deste material nos permite deduzir que o efeito protetor cerebral da nimodipina reduziu de $76,4 \%$ os eventos.

Aprofundando a análise do subgrupo de pacientes com sintomas neurológicos, encontramos que, no Grupo nimodipina, os pacientes que apresentaram quadro neurológico (todos reversíveis), eram de operação complexa com tempo de perfusão extracorpórea duas vezes maior que no Grupo placebo (tempo de extracorpórea no Grupo nimodipina $127+/-46,8$ contra $61,71+/-15,13$ minutos no Grupo placebo). Por outro lado, no mesmo material, no Grupo placebo observamos quadros neurológicos tanto permanentes quanto reversíveis em pacientes com revascularização do miocárdio isolada (sem diferenças entre os subgrupos nas variáveis bioquímicas, pO2, pCO2 e glicemia). Estes dados sugerem um efeito protetor da nimodipina.

A maior permanência dos pacientes que receberam nimodipina na UTI $(180+/-135$ horas contra $52+/-13$ horas para o placebo) e na enfermaria $(13,5+/-4,95$ dias contra $6,5+/-0,5$ para o placebo) foi conseqüência da maior complexidade cirúrgica. A mortalidade foi similar em ambos os grupos, um óbito em cada, sendo que o do grupo placebo foi por morte súbita, no dia da alta e no grupo nimodipina foi por choque cardiogênico no paciente que infartou em sala e evolui com baixo débito.

A incidência geral de eventos neurológicos foi de $9,4 \%$ (6 em 64 ), sendo que no Grupo nimodipina foi de $10 \%(3 / 30)$ e no Grupo placebo de $17,64 \%$ (6/34). Considerando o AVC com seqüela o único caso no Grupo placebo de uma incidência de $2,94 \%$ (1/34) contra zero no Grupo nimodipina. Os achados no Grupo placebo são compativeis com as referências bibliográficas.

Em resumo, considerando as características clínico-cirúrgicas dos pacientes do Grupo nimodipina, podemos afirmar que representavam um risco neurológico maior que o Grupo placebo; mesmo assim, a incidência global de eventos neurológicos 
Manrique R, Pavanello R, Magalhães HM - Emprego da nimodipina (oxigen) como protetor cerebral na cirurgia de revascularização do miocárdio com circulação extracorpórea em pacientes idosos.

Rev Bras Cir Cardiovasc 1996; 11 (4):248-58

no Grupo nimodipina foi $76,4 \%$ menor, o que é um forte indício de proteção neuronal.

Ultimamente, os antagonistas do cálcio têm sido responsabilizados por graves efeitos colaterais (14).

O trabalho de LEGAULT et al. (15) chamou a nossa atenção, não só pela semelhança nos objetivos, mas também pela diferença nos seus resultados quando comparados com os nossos. Trata-se de um estudo da neuroproteção da nimodipina em pacientes submetidos a cirurgia cardíaca com circulação extracorpórea. Esta pesquisa foi interrompida precocemente por uma elevada mortalidade no grupo que recebeu nimodipina (Tabela 16).

Os autores encontraram uma incidência proibitiva de sangramento incoercível, que, em forma direta ou indireta imediata, foi responsável pela elevada mortalidade. Relatam uma incidência de $13,3 \%$ de hemorragia grave (drenagem acima de $2400 \mathrm{ml} \mathrm{em}$ 24 horas) nos pacientes tratados com nimodipina, contra $4,1 \%$ no Grupo placebo (Tabela 17).

Em nossa observação, utilizando a mesma definição de hemorragia severa, sangramento acima de $2400 \mathrm{ml}$ em 24 horas, encontramos 1 paciente no Grupo nimodipina (drenagem de $2960 \mathrm{ml}$ ) e nenhum no Grupo placebo (o máximo volume drenado foi de $2030 \mathrm{ml}$ ) Nenhum destes pacientes foi a óbito.

Em nenhum de nossos casos foi utilizado concentrado de fatores pró-coagulantes ou de plaquetas, para coibir drenagens maiores.

LEGAULT et al (15) consideram o uso de antagonistas de cálcio em geral e nimodipina em especial, como causador de hemorragias com alta probabilidade de óbito.

Os autores lançam várias hipóteses, para explicar, as hemorragias com tão elevada mortalidade. A principal é o efeito antiplaquetário dos antagonistas do cálcio; porém, no trabalho não mostram nenhuma evidência de que este seja o mecanismo específico nos seus pacientes; em outras palavras, não encontramos nenhum exame de função plaquetária com resultados que confirmem esta opinião. Consideramos que a gravidade das complicações
TABELA 16

QUADRO COMPARATIVO DA MORTALIDADE

\begin{tabular}{lcc}
\hline MORTALIDADE & LEGAULT & HOSP. CORAÇÃO \\
\hline Periodo & até 6 meses & até 41 meses \\
Nimodipina & $8(10,7 \%)$ & $2(6 \%)$ \\
Placebo & $1(1,3 \%)$ & $5(17 \%)$ \\
\hline
\end{tabular}

justificava a incorporação destes testes, seja como parte do protocolo ou como exame diagnóstico da causa da hemorragia, ou até como informação útil para orientar o tratamento.

$\mathrm{Na}$ Tabela 17 mostramos, comparativamente, que em nossa causuística a evolução foi absolutamente diferente, não observando, no pós-operatório imediato de ambos os grupos, sangramento aumentado, nem diferença na mortalidade intra-hospitalar.

O controle pós-operatório tardio, até 41 meses, mostra maior mortalidade no Grupo placebo do que no Grupo nimodipina; porém, consideramos esta diferença sem relação causal com a medicação, e sim como expressão da história natural da própria doença coronária.

Como explicação para a diferença evolutiva entre os pacientes de LEGAULT et al. ${ }^{(15)}$ e nossa casuística, devemos considerar que aqueles autores realizaram a sua pesquisa em pacientes valvopatas. Esta população é considerada como mais propensa a hemorragias no pós-operatório imediato, possivelmente relacionadas com alterações hepáticas crônicas causadas pelas descompensações cardíacas com congestão hepática que originaria um distúrbio na síntese de fatores procoagulantes hepato-dependentes. A favor desta hipótese encontramos, no trabalho de Legault, que no subgrupo nimodipina predominaram os pacientes com história de doença pulmonar ( $23 \%$ contra $9 \%$ no subgrupo placebo) e a insuficiência cardiaca congestiva (56\% contra $38 \%$ ); ambas as diferenças estatísticamente significativas $(p=0,03)$.

O paciente coronariano, escolhido como modelo em nosso trabalho, é mais propenso a complicações vaso-oclusivas, mantendo características pró-

TABELA 17

HEMORRAGIA SEVERA E ÓBITOS CAUSADOS POR SANGRAMENTO

\begin{tabular}{|c|c|c|c|c|}
\hline \multirow{2}{*}{ 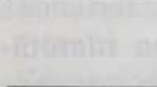 } & \multicolumn{2}{|c|}{ HEMORRAGIA SEVERA } & \multicolumn{2}{|c|}{ ÓBITOS POR HEMORRAGIA } \\
\hline & LEGAULT & HOSP. CORAÇÃO & LEGAULT & HOSP. CORAÇÃO \\
\hline Nimodipina & 10 & 1 & 5 & 0 \\
\hline Placebo & 3 & 0 & 1 & 0 \\
\hline
\end{tabular}


Manrique R, Pavanello R, Magalhães H M - Emprego da nimodipina (oxigen) como protetor cerebral na cirurgia de revascularização do miocárdio com circulação extracorpórea em pacientes idosos.

Rev Bras Cir Cardiovasc 1996; 11 (4):248-58

trombóticas. O impacto da nimodipina sobre a hemostasia nestes grupos de pacientes pode ser diferente.

Por outro lado, a preservação dos fatores de coagulação e plaquetas durante a circulação extracorpórea depende, entre outros, do efeito da heparina. De acordo com nosso esquema, a dose inicial deve prolongar o TCA até 600 segundos. Este valor é mantido durante todo o período extracorpóreo, se necesário, com doses suplementares de heparina. Em nossa opinião, este protocolo evita a deterioração de fatores de coagulação plasmáticos e plaquetas (16).

Uma terceira opção é a interação medicamentosa. O paciente cardiológico geralmente é tratado com multiplos fármacos. Por esta razão, no planejamento de nosso trabalho excluímos os pacientes em uso de drogas com eventual interferência no efeito vaso-dilatador e plaquetário, com os antagonistas do cálcio. Infelizmente, por falta de informação, não podemos avaliar se Legault et al. tiveram o mesmo cuidado.

Aprofundando as diferenças com os resultados daqueles autores ${ }^{(15)}$, nosso trabalho permite definir uma tendência benéfica no uso de nimodipina na prevenção de eventos neurológicos. Legault et al. não acham nenhuma vantagem no uso de nimodipina. Postulamos, como explicação, que essa falta de efeito protetor, no trabalho de Legault, está relacionada com a dose e via de administração da nimodipina. Para otimizar o efeito profilático de uma droga necessitamos obter níveis efetivos desde a fase pré-operatória e, fundamentalmente, na intra e na pós-operatória imediata e, por esta razão, utilizamos a via endovenosa. Pensamos que a dose proposta e a via de administração escolhidas por Legault et al. não são ideais para garantir o efeito neuro-protetor da nimodipina, neste protocolo específico. A via oral no intra e no pós-operatório não oferece condições de absorção normal, pela influência da anestesia e da circulação extracorpórea na função gastrintestinal. Nosso protocolo prevê o uso de nimodipina endovenosa para atingir um máximo efeito neste curto período e absoluta independência de interferências gastrintestinais; em conseqũência, observamos uma diferença entre o Grupo nimodipina em relação ao Grupo placebo, mesmo considerando que a nossa casuística é de idosos com coronariopatia isquêmica $e$, freqüentemente, com problemas vasculares em outros territórios. Por razões de rigor científico, qualificamos esta diferença apenas como uma tendência devido ao tratamento da nossa amostra.

\section{RBCCV 44205-316}

Manrique R, Pavanello R, Magalhães H M - Use of nimodipine for cerebral injury prophylaxis in surgical myocardial revascularization with extracorporeal circulation in elderly patients. Rev Bras Cir Cardiovasc $1996 ; 11(4): 248-58$.

ABSTRACT: Around 5 percent of the patients submitted to heart surgery with extracorporeal circulation present neurological problems. In relation to neuropsicological functions the alteration rate achieve 50 to $70 \%$. An independent variable to stroke is age, the frequency in patients older then 75 years is close to $9 \%$. Taking in account that rate of the elderly population grows very fast and that the coronary patient are frequently older than 65 years, we found very important to search for prophylactic ways to reduce this incidence. New drugs promise to achieve the goal of a prophylaxis of the cerebral injury induced by extracorporeal circulation and nimodipine, a neurovascular specific calcium channel blocker, seems to be a great promise for the prevention of cerebral damage. This is a pilot, double blind, randomized and controlling study with 64 patients, 30 in the nimodipine group and 34 in the placebo branch. Demographics and preoperative data were similar, without any significative difference but the incidence of previous transitory ischaemic attack. The nimodipine group shows a reduction in the number of neurological events in comparation with the placebo group, although a most complicated surgical procedure and inter and post-operative evolution. In the nimodipine group 3 patients presented mental confusion but in the placebo branch we found also 3 cases of mental confusion plus 2 cases of drowsy and one ischaemic stroke with sequels. In summary in the nimodipine group were 3 cases in 30 patients $(10 \%)$ against 6 in 34 patients (17.64\%) in une placebo group. It means a neurological events reduction of $76.4 \%$. Legault reported high mortality in patients using nimodipine in cardiac valve replacement surgery, mainly related to bleeding. We don't found such correlation. The hospital - mortality was similiar in both groups (1 patient in each group) and non statistically significant difference in bleeding (1 severe case in the nimodipine group). In the follow up till 41 months there was a higher mortality in the placebo (4 patients) than in the nimodipine group (1 case).

DESCRIPTORS: Myocardial revascularization. Cerebral ischemia, prevention and control. Confusion, prevention and control. Nimodipine, therapeutic use. Nimodipine, administration and dosage. Myocardial revacularization, mortality. Extracorporeal circulation, adverse effects. Double-blind method. 
Manrique R, Pavanello R, Magalhães H M - Emprego da nimodipina (oxigen) como protetor cerebral na cirurgia de revascularização do miocárdio com circulaçāo extracorpórea em pacientes idosos.

Rev Bras Cir Cardiovasc 1996; 11 (4):248-58 .

\section{REFERÊNCIAS BIBLIOGRÁFICAS}

1 Mills S A - Cerebral injury and cardiac surgery. Ann Thorac Surg 1993; 56 (Supl.5): 586-91.

2 Breuer A C, Furlan A J, Hanson M R et al. - Central nervous system complications of coronary bypass graf surgery: prospective analysis of 421 patients. Stroke 1983; 14: 682-7.

3 Shau P J, Bats D, Cartridge NE F et al. - Neurologic and neuropsychologic morbidity following major surgery: comparation of coronary artery bypass and peripheral vascular surgery. Stroke 1987; 32: 700-7.

4 Weintraub W S, Jones E L, Craver J, Guyton R, Cohen C - Determinants of prolonged length of hospital stay after coronary bypass surgery. Circulation 1982; 80: 276-84.

5 Toward R \& Kazda S - The cellular mechanism of action of nimodipine. Br J Pharmacol 1979; 67: 409-10.

6 Brand L, Anderson K E, Edvinson L, Ljunggren B Effects of extracellular calcium and of calcium antagonist on the contractil response of isolated human pial and mesenteric arteries. J Cereb Blood Flow Metab 1981; 1: 339-47.

7 Harper A M - Effects of dihydropyridine - type calcium antagonist on cerebral flow and metabolism. In: Fleckenstein A, Breemen C, Grob R, Hoffmeister F, eds. Cardiovascular effects of dihydropyridine type calcium antagonist and agonist. Berlin-Heidelberg: Sprirger-Verlag, 1985; 353-60.

8 Siesg B K - Cell damage in the brain: a speculative synthesis. J Cereb Blood Flow Metab 1981; 1: 155-61.
9 Steen P A, Newbwerg L A, Milde $\mathrm{J} \mathrm{H}$, Michenfelder $\mathrm{J}$ D - Nimodipine improve cerebral flow and neurologic recovery after complete cerebral ischaemia in the dog. J Cereb Blood Flow Metab 1983; 3: 38-42.

10 Steen P A, Newbwerg L A, Milde $\mathrm{J} \mathrm{H}$, Michenfelder $\mathrm{J}$ $D$ - Cerebral flow and neurologic outcome when nimodipine is given after complete cerebral ischaemia in the dog. J Cerebral Blood Flow Metab 1984; 4: 82-5.

11 Steen P A, Gisvold S E, Milde J H et al. - Nimodipine improves outcome when given after complete ischaemia in primates. Anesthesiology 1985; 62: 406-10.

12 Caettaneo A D - Protezione cerebrale. Minerva Anestesiol 1993; 59: 403-17.

13 Forsman M, Olsnes B J, Sem B G, Steen P A - Effects of nimodipine on cerebral blood flow and neuropsychological outcome after cardiac surgery. $\mathrm{Br} J$ Anesthesia 1990; 65: 514-20.

14 Furberg C D, Psaty B M, Meyer J V - Nifedipine: doserelated increase in mortality in patients with coronary heart disease. Circulation 1995; 92 : 1326-31.

15 Legault C, Furberg C, Wagenknecht L E et al. Nimodipine neuroprotection in cardiac valve replacement. Stroke 1996; 27: 593-8.

16 Manrique R - Monitoring of platelet function in the cardiovascular surgery patient. In: Pifarré R. Anticoagulation, hemostasis and blood preservation in cardiovascular surgery. St. Louis: Hanley \& Belfus Inc., 1993: 201-13. 